\title{
Capacity Analysis of a Decode-and-Forward Cooperative Network under Adaptive Transmission
}

\author{
Prasanna Kalansuriya and Chintha Tellambura \\ Department of Electrical and Computer Engineering \\ University of Alberta, Edmonton, Alberta, Canada T6G 2V4 \\ Email: \{kalansur, chintha\}@ece.ualberta.ca
}

\begin{abstract}
This paper presents a capacity analysis of a decode-and-forward (DF) cooperative network with adaptive source transmission. Three different adaptive techniques are considered: (i) optimal simultaneous power and rate adaptation (OPRA); (ii) constant power with optimal rate adaptation (ORA); (iii) truncated channel inversion with fixed rate (TIFR). Closed form capacity expressions are derived for non-identical and independently distributed (non-i.i.d.) Nakagami- $m$ fading wireless environments. These expressions are validated by simulation results obtained through Monte Carlo simulation. It is observed that OPRA and ORA provide roughly the same capacity, while TIFR yields slightly less capacity relative to the other two.
\end{abstract}

\section{INTRODUCTION}

Adaptive transmission schemes are employed in wireless communication systems along with other techniques to achieve high throughput in time-varying channels. The use of adaptive techniques in conventional communication systems have been widely studied by researchers [1]-[5]. The basic idea behind the adaptive approach is to dynamically vary the transmission parameters such as the symbol rate, modulation scheme, power level, coding rate/scheme according to the quality of the channel, as opposed to the use of a fixed set of parameters suited for the worse case channel conditions.

Cooperative diversity is a technique used to achieve spatial diversity through cooperation of a set of relay nodes. The use of adaptive transmission schemes in cooperative networks has recently received some attention. The performance of a single relay cooperative network with adaptive modulation was studied in [6] where the author considered both amplify-and-forward (AF) and decode-andforward (DF) relays in a Rayleigh fading wireless environment to obtain capacity expressions under three adaptive transmission schemes: (i) optimal simultaneous power and rate adaptation (OPRA) (ii) constant power with optimal rate adaptation (ORA) (iii) truncated channel inversion with fixed rate (TIFR). The recent work of Nechiporenko et al. [7] has extended work of [6] to an $N \geq 1$ AF relay network. In [8] Nechiporenko et al. extended [7] by considering optimum switching thresholds for adaptive modulation.

This paper provides a capacity analysis of a DF cooperative network with adaptive transmission. The channel 978-1-4244-3508-1/09/\$25.00 (C2009 IEEE

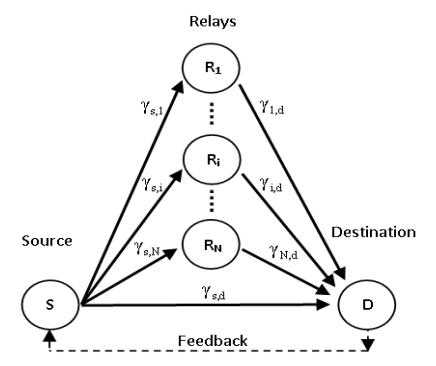

Fig. 1. Relay network with adaptive transmission.

capacity is for the three adaptive transmission schemes OPRA, ORA and TIFR and derived for independent and non-identically distributed (non-i.i.d.) Nakagami- $m$ wireless channels. The effect of a decoding threshold in DF relays on the achievable channel capacity is also analyzed. Our work is an extension of the previous work of [6], [7]. In [6] the direct path between source and destination is not considered and in [7] only the performance of AF relays, but not DF relays, were investigated.

The rest of the paper is organized as follows. Section II presents the channel and system model. Capacity analysis is conducted in Section III. The numerical results are presented in Section IV. Conclusions are given in Section V.

\section{Notations}

$\mathbb{Z}$ is the set of integers and $\mathbb{Z}^{+}$is the set of positive integers. $M_{X}(s)$ is the moment generating function (MGF) of random variable $X$ and is defined as $M_{X}(s)=\mathbf{E}\left(e^{-s X}\right)$ where $\mathbf{E}(\cdot)$ is the expectation operator.

\section{Channel and System Model}

Fig. 1 depicts the system model of the DF cooperative network. Source $S$ transmits to destination $D$, assisted by $N$ cooperating parallel $\mathrm{DF}$ relays. The communication protocol involves two phases. During the first phase, source transmits its message which is received by the destination and relays $R_{i}, i \in\{1,2, \cdots N\}$, due to the broadcast nature of the wireless channel. In the second phase, each of the relays decodes and retransmits a freshly encoded 


$$
\begin{gathered}
f_{\gamma_{t o t}}\left(\gamma \mid \underline{\mathbf{b}}_{k}\right)=\sum_{j=1}^{m_{s, d}} \frac{\Delta_{j}\left(\underline{\mathbf{b}}_{k}\right)}{(j-1) !}\left(\frac{m_{s, d}}{\bar{\gamma}_{s, d}}\right)^{j} \gamma^{j-1} e^{-\frac{m_{s, d} \gamma}{\bar{\gamma}_{s, d}}}+\sum_{i=1, b_{i} \neq 0}^{N} \sum_{j=1}^{m_{i, d}} \frac{\Psi_{i, j}\left(\underline{\mathbf{b}}_{k}\right)}{(j-1) !}\left(\frac{m_{i, d}}{\bar{\gamma}_{i, d}}\right)^{j} \gamma^{j-1} e^{-\frac{m_{i, d} \gamma}{\bar{\gamma}_{i, d}}} \\
\Delta_{i}\left(\underline{\mathbf{b}}_{k}\right)=\frac{\left(\frac{\bar{\gamma}_{s, d}}{m_{s, d}}\right)^{\left(i-m_{s, d}\right)}}{\left(m_{s, d}-i\right) !} \frac{\partial^{m_{s, d}-i}}{\partial s^{m_{s, d}-i}}\left[\prod_{j=1, b_{j} \neq 0}^{N}\left(1+\frac{\bar{\gamma}_{j, d}}{m_{j, d}} s\right)^{-m_{j, d}}\right]_{s=-\frac{m_{s, d}}{\bar{\gamma}_{s, d}}} \\
\Psi_{i, j}\left(\underline{\mathbf{b}}_{k}\right)=\frac{\left(\frac{\bar{\gamma}_{i, d}}{m_{i, d}}\right)^{\left(j-m_{i, d}\right)}}{\left(m_{i, d}-j\right) !} \frac{\partial^{m_{i, d}-j}}{\partial s^{m_{i, d}-j}}\left[\left(1+\frac{\bar{\gamma}_{s, d}}{m_{s, d}} s\right)^{-m_{s, d}}\left[\prod_{l=1, l \neq i, b_{l} \neq 0}^{N}\left(1+\frac{\bar{\gamma}_{l, d}}{m_{l, d}} s\right)^{-m_{l, d}}\right]\right]_{s=-\frac{m_{i, d}}{\bar{\gamma}_{i, d}}}
\end{gathered}
$$

version of the received signal to the destination by using orthogonal transmission channels [9]. The received signals at the destination are combined using a diversity combining technique, which also mitigates the effect of fading.

Channel State Information (CSI) estimated at the receiver is sent to the source transmitter through a reliable low data rate low delay feedback as shown in Fig. 1. The transmitter uses this CSI to adapt its transmission to the changing channel conditions.

The DF relays operate using an adaptive policy [9]-[11] where relays would decode received signals from the source if they are above a particular instantaneous signal to noise ratio (SNR). This threshold $\mathrm{SNR}$ value, $\gamma_{T}$, is the minimum SNR that would guarantee successful decoding at the DF relay. $\gamma_{T}=2^{R(N+1)}-1$ where $R$ is the target information rate below which the $\mathrm{DF}$ relay would fail to decode the source message. Under this scheme, the number of relays forwarding the source message is a random variable. If one complete cycle of cooperation in the network is defined as a state of the network, then the DF relays operating in a particular state $k$ can be represented using a vector $\underline{\mathbf{b}}_{k}$

$$
\begin{aligned}
& \underline{\mathbf{b}}_{k}=\left(b_{N}, b_{N-1}, \ldots, b_{i}, \ldots, b_{2}, b_{1}\right), k=0,1, \ldots, 2^{N}-1 \\
& \text { and } k=2^{N-1} b_{N}+2^{N-2} b_{N-1}+\ldots+2 b_{2}+b_{1},
\end{aligned}
$$

where $b_{i}$ 's are indicator random variables taking values 1 or 0 to indicate whether the $i^{\text {th }}$ relay is forwarding source message to destination or not. $\operatorname{Pr}\left(b_{i}=1\right)=p_{i}$ and $\operatorname{Pr}\left(b_{i}=\right.$ $0)=1-p_{i}$ where $p_{i}$ is the probability that received SNR at $R_{i}$ is greater than $\gamma_{T}$. In a given cycle of cooperation the system can be in one of $2^{N}$ states described by a particular vector $\underline{\mathbf{b}}_{k}$ with a probability $\operatorname{Pr}\left(\underline{\mathbf{b}}_{k}\right)$ where

$$
\operatorname{Pr}\left(\underline{\mathbf{b}}_{k}\right)=\prod_{i=1}^{N} p_{i}^{b_{i}}\left(1-p_{i}\right)^{\left(1-b_{i}\right)} .
$$

The number of DF relays forwarding source signal to the destination is $N\left(\underline{\mathbf{b}}_{k}\right)=\sum_{i=1}^{N} b_{i}$. Maximal ratio combining (MRC) is used to combine the $N\left(\underline{\mathbf{b}}_{k}\right)+1$ orthogonally received signals at the destination which results in a total received SNR $\gamma_{t o t}=\gamma_{s, d}+\sum_{i=1}^{N} b_{i} \gamma_{i, d}$ where $\gamma_{s, d}$ and $\gamma_{i, d}$ are the instantaneous SNR values received at $D$ due to $S$ and $R_{i}$ respectively. It is assumed that the wireless environment is slowly-varying and flat-fading where the rate of change of the channel is much slower than the symbol rate. This assumption is required for the adaptation of the transmission parameters, based on the receiver feedback, to be viable. The wireless multipath fading environments between all nodes are modeled as non-i.i.d. Nakagami- $m$ fading channels. The Nakagami fading parameters between $S$ and $R_{i}, S$ and $D$, and $R_{i}$ and $D$ are $m_{s, i}, m_{s, d}$ and $m_{i, d}$ respectively. This results the probability density function of the instantaneous received SNR to be Gamma distributed.

The received SNR at the $i^{\text {th }}$ relay $R_{i}$ is $\gamma_{s, i}$ and it is Gamma distributed. Probability of relay $R_{i}$ forwarding source message to destination is

$$
p_{i}=\operatorname{Pr}\left(\gamma_{s, i}>\gamma_{T}\right)=\frac{\Gamma\left(m_{s, i}, \frac{m_{s, i} \gamma_{T}}{\bar{\gamma}_{s, i}}\right)}{\Gamma\left(m_{s, i}\right)},
$$

where $\Gamma(a, x)=\int_{x}^{\infty} t^{a-1} e^{-t} d t$ and $\Gamma(a)=(a-1) !, a \in$ $\mathbb{Z}^{+}$. Given that the relay network is in state $k, k \in$ $\left\{0, \ldots, 2^{N}-1\right\}$, described by vector $\underline{\mathbf{b}}_{k}$, the MGF of $\gamma_{t o t}$ conditioned on $\underline{\mathbf{b}}_{k}$ can be written as

$$
M_{\gamma_{t o t}}\left(s \mid \underline{\mathbf{b}}_{k}\right)=M_{\gamma_{s, d}}(s) \prod_{i=1, b_{i} \neq 0}^{N} M_{\gamma_{i, d}}(s) \text {. }
$$

For Nakagami fading, $M_{\gamma_{s, d}}(s)=\left(1+\frac{\bar{\gamma}_{s, d}}{m_{s, d}} s\right)^{-m_{s, d}}$ and $M_{\gamma_{i, d}}(s)=\left(1+\frac{\bar{\gamma}_{i, d}}{m_{i, d}} s\right)^{-m_{i, d}}$. Hence we can rewrite (7) as

$$
M_{\gamma_{t o t}}\left(s \mid \underline{\mathbf{b}}_{k}\right)=\frac{1}{\left(1+\frac{\gamma_{s, d}}{m_{s, d}} s\right)^{m_{s, d}}} \prod_{i=1, b_{i} \neq 0}^{N} \frac{1}{\left(1+\frac{\gamma_{i, d}}{m_{i, d}} s\right)^{m_{i, d}}} .
$$

Partial fraction expansion of (8) gives

$$
M_{\gamma_{t o t}}\left(s \mid \underline{\mathbf{b}}_{k}\right)=\sum_{j=1}^{m_{s, d}} \frac{\Delta_{j}\left(\underline{\mathbf{b}}_{k}\right)}{\left(1+\frac{\bar{\gamma}_{s, d}}{m_{s, d}} s\right)^{j}}+\sum_{i=1, b_{i} \neq 0}^{N} \sum_{j=1}^{m_{i, d}} \frac{\Psi_{i, j}\left(\underline{\mathbf{b}}_{k}\right)}{\left(1+\frac{\bar{\gamma}_{i, d}}{m_{i, d}} s\right)^{j}} .
$$

Laplace inversion of (9) gives the conditional PDF, (1), of the total received SNR at the destination conditional on $\underline{\mathbf{b}}_{k}$, $f_{\gamma_{t o t}}\left(\gamma \mid \underline{\mathbf{b}}_{k}\right) . \Delta_{i}\left(\underline{\mathbf{b}}_{k}\right)$ is given in (2) for $k \neq 0$ with $\Delta_{i}\left(\underline{\mathbf{b}}_{0}\right)=$ 0 for $i \neq m_{s, d}$ and $\Delta_{i}\left(\underline{\mathbf{b}}_{0}\right)=1$ for $i=m_{s, d} . \Psi_{i, j}\left(\underline{\mathbf{b}}_{k}\right)$ is defined in (3) for $k \neq 0$ with $\Psi_{i, j}\left(\underline{\mathbf{b}}_{0}\right)=0 \forall i, j \in \mathbb{Z}$.

By using the theorem of total probability, the PDF of total 


$$
\begin{gathered}
C_{\text {opra }}=\frac{B}{\ln 2} \sum_{k=0}^{2^{N}-1} \frac{\operatorname{Pr}\left(\underline{\mathbf{b}}_{k}\right)}{\left(1+N\left(\underline{\mathbf{b}}_{k}\right)\right)}\left[\sum_{j=1}^{m_{s, d}} \frac{\Delta_{j}\left(\mathbf{b}_{k}\right)}{(j-1) !}\left(\frac{m_{s, d} \gamma_{0}}{\bar{\gamma}_{s, d}}\right)^{j} \mathcal{J}_{j}\left(\frac{m_{s, d} \gamma_{0}}{\bar{\gamma}_{s, d}}\right)+\sum_{i=1, b_{i} \neq 0}^{N} \sum_{j=1}^{m_{i, d}} \frac{\Psi_{i, j}\left(\underline{\mathbf{b}}_{k}\right)}{(j-1) !}\left(\frac{m_{i, d} \gamma_{0}}{\bar{\gamma}_{i, d}}\right)^{j} \mathcal{J}_{j}\left(\frac{m_{i, d} \gamma_{0}}{\bar{\gamma}_{i, d}}\right)\right] \\
\quad \sum_{k=0}^{2^{N}-1} \operatorname{Pr}\left(\underline{\mathbf{b}}_{k}\right)\left\{\sum_{j=1}^{m_{s, d}} \frac{\Delta_{j}\left(\underline{\mathbf{b}}_{k}\right)}{(j-1) !}\left[\frac{\Gamma\left(j, \frac{m_{s, d} \gamma_{0}}{\bar{\gamma}_{s, d}}\right)}{\gamma_{0}}-\frac{\Gamma\left(j-1, \frac{m_{s, d} \gamma_{0}}{\bar{\gamma}_{s, d}}\right)}{\left(\frac{\bar{\gamma}_{s, d}}{m_{s, d}}\right)}\right]\right. \\
\left.\quad+\sum_{i=1, b_{i} \neq 0}^{N} \sum_{j=1}^{m_{i, d}} \frac{\Psi_{i, j}\left(\underline{\mathbf{b}}_{k}\right)}{(j-1) !}\left[\frac{\Gamma\left(j, \frac{m_{i, d} \gamma_{0}}{\gamma_{i, d}}\right)}{\gamma_{0}}-\frac{\Gamma\left(j-1, \frac{m_{i, d}}{\bar{\gamma}_{i, d}}\right)}{\left(\frac{\gamma_{i, d}}{m_{i, d}}\right)}\right]\right\}=1 \\
P_{\text {out }=}=\sum_{k=0}^{2^{N}-1} \operatorname{Pr}\left(\underline{\mathbf{b}}_{k}\right)\left[\sum_{j=1}^{m_{s, d}} \frac{\Delta_{j}\left(\underline{\mathbf{b}}_{k}\right)}{(j-1) !} \Gamma\left(j, \frac{m_{s, d} \gamma_{0}}{\bar{\gamma}_{s, d}}\right)+\sum_{i=1, b_{i} \neq 0}^{N} \sum_{j=1}^{m_{i, d}} \frac{\Psi_{i, j}\left(\underline{\mathbf{b}}_{k}\right)}{(j-1) !} \Gamma\left(j, \frac{m_{i, d} \gamma_{0}}{\bar{\gamma}_{i, d}}\right)\right] \\
C_{\text {ora }}=\frac{B}{\ln 2} \sum_{k=0}^{2^{N}-1} \frac{P\left(\underline{\mathbf{b}}_{k}\right)}{\left(1+N\left(\underline{\mathbf{b}}_{k}\right)\right)}\left[\sum_{j=1}^{m_{s, d}} \frac{\Delta_{j}\left(\underline{\mathbf{b}}_{k}\right)}{(j-1) !}\left(\frac{m_{s, d}}{\bar{\gamma}_{s, d}}\right)^{j} \mathcal{I}_{j}\left(\frac{m_{s, d}}{\bar{\gamma}_{s, d}}\right)+\sum_{i=1, b_{i} \neq 0}^{N} \sum_{j=1}^{m_{i, d}} \frac{\Psi_{i, j}\left(\underline{\mathbf{b}}_{k}\right)}{(j-1) !}\left(\frac{m_{i, d}}{\bar{\gamma}_{i, d}}\right)^{j} \mathcal{I}_{j}\left(\frac{m_{i, d}}{\bar{\gamma}_{i, d}}\right)\right]
\end{gathered}
$$

received SNR $\gamma_{t o t}$ unconditional on $\underline{\mathbf{b}}_{k}$ can the be written in terms of (5) and (1) as

$$
f_{\gamma_{\text {tot }}}(\gamma)=\sum_{k=0}^{2^{N}-1} f_{\gamma_{t o t}}\left(\gamma \mid \underline{\mathbf{b}}_{k}\right) \operatorname{Pr}\left(\underline{\mathbf{b}}_{k}\right) .
$$

\section{Capacity Analysis Under Adaptive TRANSMISSION}

This section provides the closed form expressions for capacity under three distinct adaptive transmission schemes, namely OPRA, ORA and TIFR.

\section{A. Optimal Simultaneous Power and Rate Adaptation (OPRA)}

In this scheme the source transmit power $S(\gamma)$ is varied according to the received SNR at the destination under an average power constraint $\int_{0}^{\infty} S(\gamma) f_{\gamma_{\text {tot }}}(\gamma) d \gamma=S$, [1], [2]. The channel capacity is achieved when the adaptation policy of $S(\gamma)$ is a water filling formula in time [12]. Under this scheme the channel capacity $C_{\text {opra }}$ (in bits/second) is given by [1], [2]

$$
C_{\text {opra }}=\frac{B}{(N+1) \ln 2} \int_{\gamma_{0}}^{\infty} \ln \left(\frac{\gamma}{\gamma_{0}}\right) f_{\gamma_{t o t}}(\gamma) d \gamma,
$$

where $B$ (in Hertz) is the bandwidth of the channel and $\gamma_{0}$ is the optimal cutoff SNR below which the transmission is stopped. Since the transmission takes place in $(N+1)$ orthogonal channels, a normalizing factor of $1 /(N+1)$ is used in (15)

This capacity $C_{\text {opra }}$ is attained by adapting power and rate simultaneously using the perfect channel state information available at both the source transmitter and destination receiver. Substituting (1) into (15), and making use of the theorem of total probability to average the capacity over all the states of the network, the closed-form expression for $C_{\text {opra }}$ can be derived (10). Here $N\left(\underline{\mathbf{b}}_{k}\right)$ is the number of DF relays forwarding the source signal to the destination for a given state of the network specified by the vector $\underline{\mathbf{b}}_{k}$. The integral $\mathcal{J}_{n}(\mu)=\int_{1}^{\infty} t^{n-1} \ln (t) e^{-\mu t} d t, \mu>0 ; n \in \mathbb{Z}^{+}$, is evaluated in closed-form in [2, eq. (70)].

The optimal cutoff SNR $\gamma_{0}$ below which the transmission is halted satisfies

$$
\int_{\gamma_{0}}^{\infty}\left(\frac{1}{\gamma_{0}}-\frac{1}{\gamma}\right) f_{\gamma_{t o t}}(\gamma) d \gamma=1
$$

and $\gamma_{0}$ is found by solving numerically for $\gamma_{0}$ in (16). By substituting for $f_{\gamma_{t o t}}(\gamma),(16)$ can be expressed as (11).

As the transmission is halted when $\gamma_{t o t}<\gamma_{0}$, an outage event occurs when the received SNR falls below the optimal threshold $\gamma_{0}$. This outage probability is given in (12).

\section{B. Optimal Rate Adaptation with Constant Transmit Power (ORA)}

In ORA scheme the source transmit power is kept constant while the transmission rate is adapted according to channel state information at the receiver. Capacity under this scheme is simply the ergodic capacity averaged over the distribution of the received SNR. For ORA, the channel capacity $C_{\text {ora }}$ is given by [1], [2]

$$
C_{\text {ora }}=\frac{B}{(N+1) \ln 2} \int_{0}^{\infty} \ln (1+\gamma) f_{\gamma_{t o t}}(\gamma) d \gamma .
$$

Substituting (1) into (17), and making use of the integral $\mathcal{I}_{n}(\mu)=\int_{0}^{\infty} t^{n-1} \ln (1+t) e^{-\mu t} d t, \mu>0 ; n \in \mathbb{Z}^{+}$, which can be evaluated in a closed-form as in [2, eq.(78)], and the theorem of total probability, the closed-form expression for the capacity $C_{\text {ora }}$ is derived to be (13).

\section{Channel Inversion with Fixed Rate (TIFR)}

In this scheme the transmitter only adjusts the transmit power level to provide a constant SNR at the destination. At the transmitter the power level is adapted proportional to the inverse of the channel gain as long as the received 


$$
\begin{aligned}
C_{\text {tifr }}= & \frac{B}{\ln 2} \sum_{k=0}^{2^{N}-1} \frac{\operatorname{Pr}\left(\underline{\mathbf{b}}_{k}\right)}{\left(1+N\left(\underline{\mathbf{b}}_{k}\right)\right)} \ln \left[1+\left\{\sum_{j=1}^{m_{s, d}} \frac{\Delta_{j}\left(\underline{\mathbf{b}}_{k}\right)}{(j-1) !}\left(\frac{m_{s, d}}{\bar{\gamma}_{s, d}}\right) \Gamma\left(j-1, \frac{m_{s, d} \hat{\gamma}_{0}}{\bar{\gamma}_{s, d}}\right)+\sum_{i=1, b_{i} \neq 0}^{N} \sum_{j=1}^{m_{i, d}} \frac{\Psi_{i, j}\left(\underline{\mathbf{b}}_{k}\right)}{(j-1) !}\left(\frac{m_{i, d}}{\bar{\gamma}_{i, d}}\right)\right.\right. \\
& \left.\left.\times \Gamma\left(j-1, \frac{m_{i, d} \hat{\gamma}_{0}}{\bar{\gamma}_{i, d}}\right)\right\}^{-1}\right]\left[\sum_{j=1}^{m_{s, d}} \frac{\left.\Delta_{j} \underline{\mathbf{b}}_{k}\right)}{(j-1) !} \Gamma\left(j, \frac{m_{s, d} \hat{\gamma}_{0}}{\bar{\gamma}_{s, d}}\right)+\sum_{i=1, b_{i} \neq 0}^{N} \sum_{j=1}^{m_{i, d}} \frac{\Psi_{i, j}\left(\underline{\mathbf{b}}_{k}\right)}{(j-1) !} \Gamma\left(j, \frac{m_{i, d} \hat{\gamma}_{0}}{\bar{\gamma}_{i, d}}\right)\right] .
\end{aligned}
$$

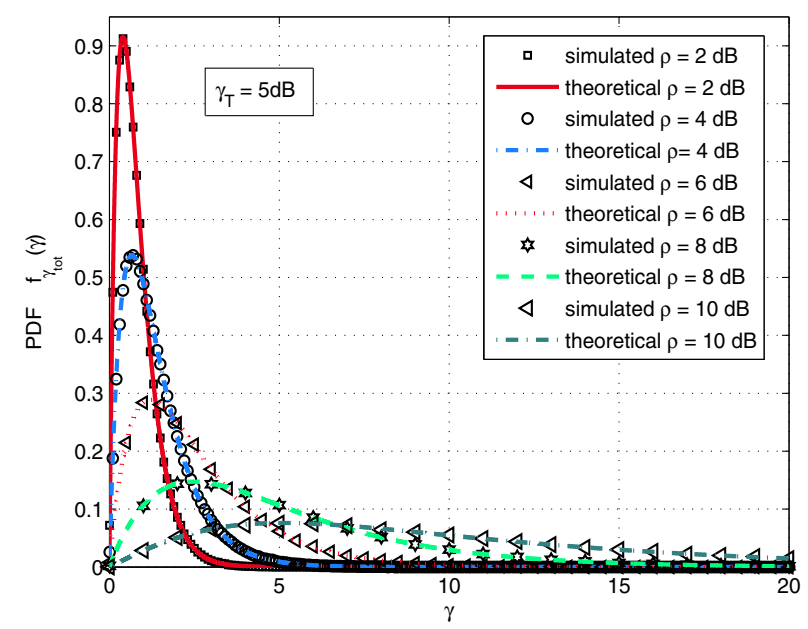

Fig. 2. PDF of the total received SNR $f_{\gamma_{t o t}}(\gamma)$ at destination for different transmit SNR values $\left(\gamma_{T}=5 \mathrm{~dB}\right)$.

SNR is above a particular cutoff SNR level $\gamma_{0}$. Therefore this power adaptation scheme inverts the effects of channel fading making the channel appear as an AWGN channel. It is also less complex than the previous two adaptive schemes. The channel capacity $C_{\text {tifr }}$ is given by [1], [2]

$C_{\text {tifr }}=\frac{B}{(N+1) \ln 2} \ln \left(1+\left[\int_{\gamma_{0}}^{\infty} \frac{f_{\gamma_{\text {tot }}}(\gamma) d \gamma}{\gamma}\right]^{-1}\right)\left(1-P_{\text {out }}\right)$.

By substituting (1) into (19) and making use of the theorem of total probability the capacity for TIFR scheme can be expressed as (18). Here, $\hat{\gamma}_{0}$ is the optimal cutoff SNR below which transmission is suspended. $\hat{\gamma}_{0}$ for this scheme is different from $\gamma_{0}$ of OPRA scheme which is always bounded between $[0,1]$, [2]. Since transmission is stopped when the received SNR is below the cutoff SNR, this scheme also suffers an outage event similar to that of the OPRA scheme.

\section{RESUlts}

The results obtained for channel capacity and outage probability under the three different adaptive transmission schemes are compared here. The theoretical curves and semi-analytical Monte Carlo simulation results ${ }^{1}$ are presented. A two relay cooperative network with the following

\footnotetext{
${ }^{1}$ The channel is simulated statistically using random number generation and the analytical capacity equations are used to obtain results which are then averaged over a large number of iterations.
}

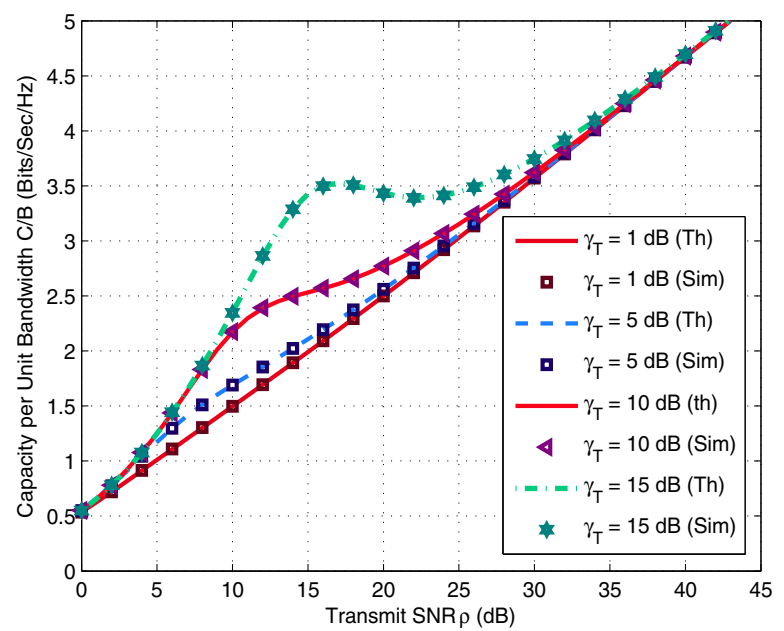

Fig. 3. Capacity comparison for the ORA scheme for varying thresholds.

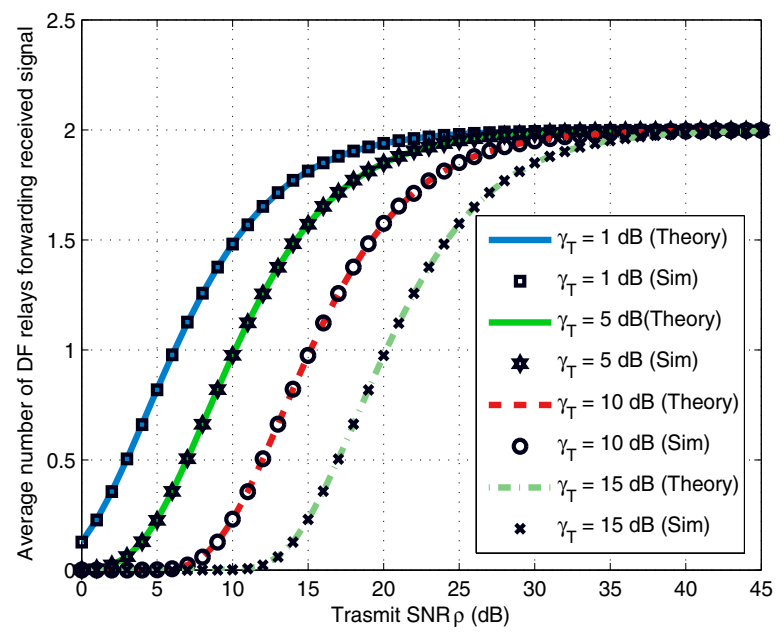

Fig. 4. Average number of DF relays forwarding received signal to destination for different $\gamma_{T}$.

channel parameters was considered in the analysis, $\bar{\gamma}_{s d}=$ $0.5 \rho, \bar{\gamma}_{s 1}=0.2 \rho, \bar{\gamma}_{s 2}=0.7 \rho, \bar{\gamma}_{1 d}=0.9 \rho, \bar{\gamma}_{2 d}=0.4 \rho$, $m_{s d}=2, m_{s 1}=1, m_{s 2}=2, m_{1 d}=3, m_{2 d}=1$, where $\rho$ is the average transmit SNR.

The PDF of the total received SNR $\gamma_{t o t}$ is shown in Fig. 2. The decoding threshold $\gamma_{T}$ is chosen to be $5 \mathrm{~dB}$ and PDF plots are obtained for different transmit SNR values near $\gamma_{T}$. 


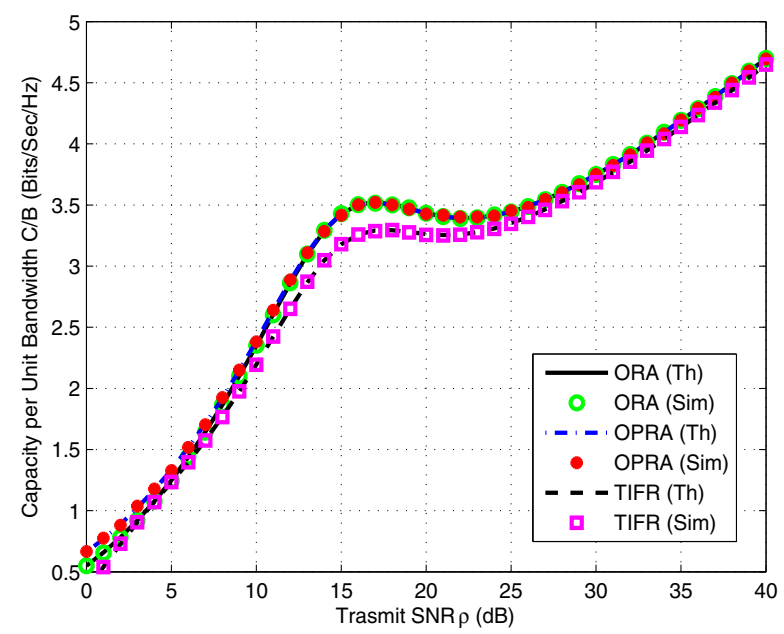

Fig. 5. Capacity comparison of different adaptive transmission schemes with a $\gamma_{T}=15 \mathrm{~dB}$ decoding threshold.

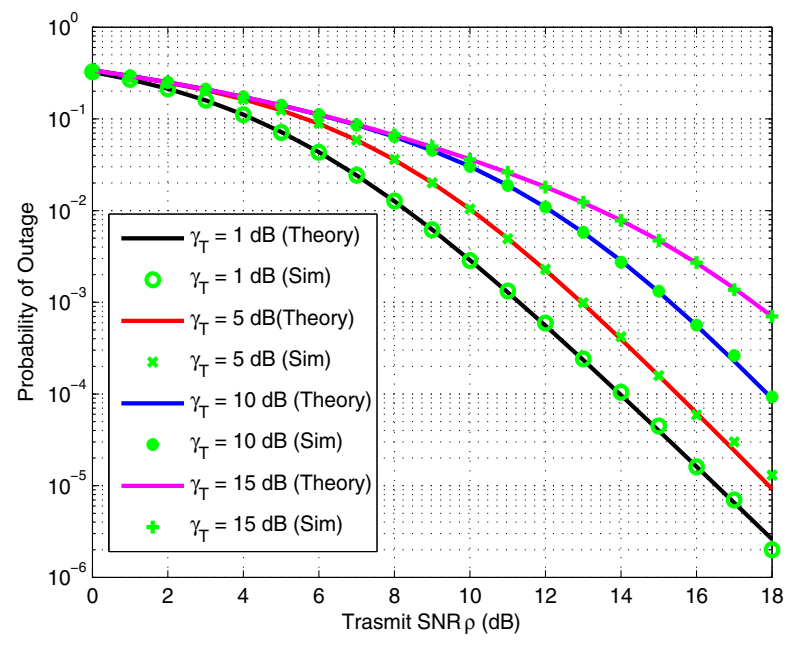

Fig. 6. Outage probability for the OPRA scheme for varying thresholds.

A large variation in the shape of PDF's can be seen around $\rho=\gamma_{T}$.

Fig. 3 shows the capacity analysis under the ORA scheme for non-i.i.d. DF relay network for varying relay decoding thresholds, $\gamma_{T}$. A local maximum is observed when $\rho$ is near the $\gamma_{T}$ value and in high SNR region the effect of $\gamma_{T}$ on capacity is negligible. This is due to the change in the average number of relays forwarding source signal to the destination, which directly effects the capacity (17).

Fig. 4 shows the variation of the average number of relays forwarding source signal, $\tilde{N}$, against $\rho$ for different $\gamma_{T}$. For $\gamma_{T}=15 \mathrm{~dB}$ on average no relays forward signals to the destination $(\tilde{N} \leq 0.5)$ until $\rho>17 \mathrm{~dB}$ and for $17 \mathrm{~dB}<\rho<$ $35 \mathrm{~dB} \tilde{N}$ gradually increases from 0.5 to 2 . This observation explains why the capacity curve for $\gamma_{T}=15 \mathrm{~dB}$ in Fig. 3

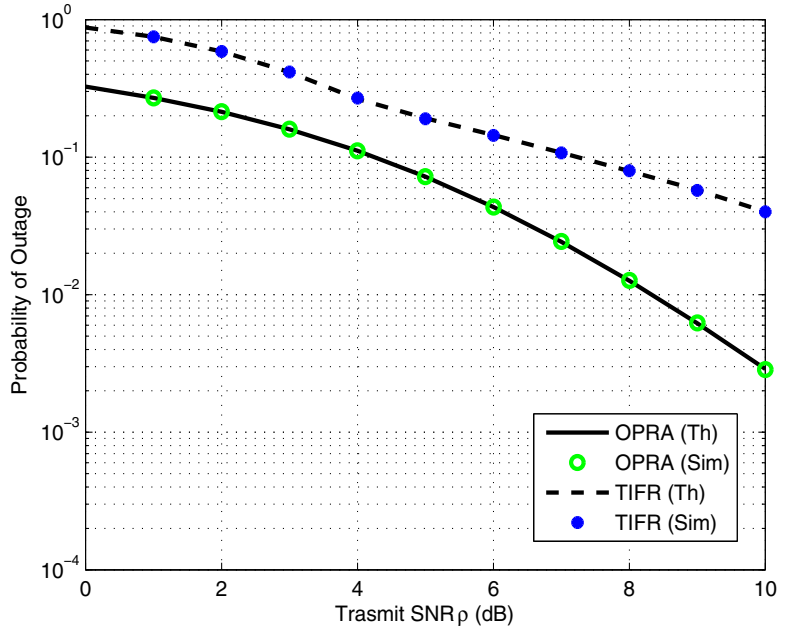

Fig. 7. Outage probability comparison of OPRA and TIFR schemes under a $\gamma_{T}=1 \mathrm{~dB}$ decoding threshold.

has a higher gradient for $0<\rho<17 \mathrm{~dB}$ followed by a local maximum and a lower gradient for $17 \mathrm{~dB}<\rho$.

The channel capacities for the three different transmission techniques are shown in Fig. 5. The results indicate that both OPRA and ORA schemes perform equally well in the high SNR region while OPRA has a marginal gain over ORA at low SNR values. TIFR provides slightly lower performance compared to OPRA and ORA.

The outage probability for OPRA is plotted in Fig. 6. The relay decoding thresholds, $\gamma_{T}$ is varied. It is clearly observed that as the decoding threshold increases the outage probability also increases. As the decoding threshold $\gamma_{T}$ increases the average number of relays available for forwarding source message to destination reduces which increases the chance of outage, hence this observation is intuitively justified. Outage probabilities of OPRA and TIFR are compared in Fig. 7. The former has better outage performance than the latter.

\section{CONCLUSION}

The capacity of Nakagami- $m$ fading channels under adaptive transmission and cooperative diversity was addressed in this paper. A DF cooperative network was considered under non-i.i.d. Nakagami- $m$ fading channels. The channel capacity expressions were derived under the three adaptive policies: (i) OPRA (ii) ORA (iii) TIFR. The OPRA and ORA schemes were found to provide better performance compared to the TIFR scheme.

\section{REFERENCES}

[1] A. J. Goldsmith and P. P. Varaiya, "Capacity of fading channels with channel side information," vol. 43, pp. 1986-1992, Nov. 1997.

[2] M.-S. Alouini and A. J. Goldsmith, "Capacity of Rayleigh fading channels under different adaptive transmission and diversitycombining techniques," vol. 48, pp. 1165-1181, Jul. 1999.

[3] A. J. Goldsmith and S.-G. Chua, "Variable-rate variable-power MQAM for fading channels," vol. 45, pp. 1218-1230, Oct. 1997. 
[4] M.-S. Alouini and A. J. Goldsmith, "Adaptive modulation over Nakagami fading channels," Wireless Personal Comm., vol. 13, pp. 119-143, 2000.

[5] R. K. Mallik, M. Z. Win, M.-S. Alouini, and A. J. Goldsmith, "Channel capacity of adaptive transmission with maximal ratio combining in correlated Rayleigh fading," vol. 3, pp. 1124-1133, Jul. 2004.

[6] M. O. Hasna, "On the capacity of cooperative diversity systems with adaptive modulation," in Wireless and Optical Communications Networks, 2005. WOCN 2005. Second IFIP International Conference on, Mar. 2005, pp. 432-436.

[7] T. Nechiporenko, K. Phan, C. Tellambura, and H. H. Nguyen, "On the capacity of Rayleigh fading cooperative systems under adaptive transmission," IEEE Transactions on : Accepted for future publication Wireless Communications.

[8] T. Nechiporenko, P. Kalansuriya, and C. Tellambura, "Performance of optimum switching adaptive M-QAM for amplify-and-forward relays," IEEE Transactions on : Accepted for future publication Vehicular Technology.

[9] J. N. Laneman, D. N. C. Tse, and G. W. Wornell, "Cooperative diversity in wireless networks: Efficient protocols and outage behavior," vol. 50, pp. 3062-3080, Dec. 2004.

[10] S. Ikki and M. Ahmed, "Performance of decode-and-forward cooperative diversity networks over Nakagami- $m$ fading channels," Global Telecommunications Conference, 2007. GLOBECOM '07. IEEE, pp. 4328-4333, Nov. 2007.

[11] N. C. Sagias, F. I. Lazarakis, G. S. Tombras, and C. K. Datsikas, "Outage analysis of decode-and-forward relaying over Nakagami- $m$ fading channels," IEEE Signal Process. Lett., vol. 15, pp. 41-44, 2008.

[12] A. Goldsmith, Wireless Communications. New York NY: Cambridge University Press, 2005. 